\section{Arturo Gárate Arias ${ }^{1}$ Vanessa Ccahuana Vásquez ${ }^{2}$ Carolina Chang Suarez ${ }^{3}$}

'Especialista en Implantología Oral. Facultad de Estomatología Roberto Beltrán. Universidad Peruana Cayetano Heredia. Lima, Perú.

Doctora en Odontología Restauradora Facultad de Estomatología Roberto Beltrán. Universidad Peruana Cayetano Heredia. Lima, Perú.

${ }^{3}$ Especialista en Periodoncia e Implantes. Facultad de Estomatología Roberto Beltrán. Universidad Peruana Cayetano Heredia. Lima, Perú

\section{Correspondencia}

Arturo Jesús, Gárate Arias

Cayo Roca Zela \# 484-Lima 33, Perú

Teléfono: (51-1) 4496329 / 993482098.

E-mail: arturoaga1@hotmail.com

\title{
Condiciones favorables para el desempeño de prótesis sobre implantes utilizando conexiones externas.
}

Gárate-Arias $\mathrm{A}^{1}$, Ccahuana-Vásquez $\mathrm{V}^{2}$, Chang $\mathrm{C}^{3}$. Condiciones favorables para el desempeño de prótesis sobre implantes utilizando conexiones externas. Rev Estomatol Herediana. 2012; 22(2):121-128

\section{RESUMEN}

La presente revisión tiene por objetivo mostrar las principales consideraciones favorables para distribuir las fuerzas de tensión a través del eje longitudinal del implante y evitar complicaciones protésicas en el sistema de conexión externa, prolongando la supervivencia o éxito del implante. Las complicaciones en el sistema de conexión externa son la fractura del pilar e implante, aflojamiento y fractura del tornillo y reabsorción ósea de la cresta alveolar. Para evitar estas complicaciones se busca una óptima precarga, estabilidad antirotacional y sentamiento pasivo. Estas propiedades son elementales para disminuir la tensión ante fuerzas de compresión, torsión y fricción. Otras consideraciones a tener en cuenta son la oclusión, el polígono de estabilización y el diseño protésico.

Palabras clave: PRECARGA, ESTABILIDAD ANTIROTACIONAL, INTERFACE IMPLANTE PILAR, HEXAGONO EXTERNO.

Favorable conditions for the performance of implant-supported prosthesis using external connection.

\section{ABSTRACT}

This review aims to show the main favorable considerations to distribute the tensile forces along the long axis of the implant and to avoid prosthetic complications focusing on the external connection system, in this way extending the implant survival or success. Complications in the external connection system are the abutment and implant fracture, screw loosening and fracture and loss of the marginal bone. In order to avoid these complications it is needed to obtain an optimal preload, antirotational stability and passive fit, which are fundamental properties for the reduction of the tension due to compression, frictional and torsion forces. Beside those, other considerations that must be taken into account are occlusion, the staggered placement of dental implants and prosthetic design.

Key Words: PRELOAD, ANTI-ROTATIONAL STABILITY, PASIVE FIT, IMPLANT ABUTMENT INTERFACE, EXTERNAL HEXAGON.

\section{Introducción}

Desde que a mediados del siglo XX el Dr. Branemark descubriera que el titanio se adhería firmemente al hueso, los implantes han sufrido una gran evolución (1). La conexión externa fue el primer sistema en ser creado, su uso ha sido el más extendido, sin embargo ha dado lugar a un importante número de complicaciones (2). Para mitigar estos problemas, la conexión externa, la conexión transmucosa y los tornillos de retención han sido sometidos a una serie de modificaciones (3).

En la actualidad existen diversos elementos que sirven para disipar la tensión a través de los componentes protésicos y sistemas de implantes, además se busca un óptimo asentamiento entre la interfase de la plataforma del implante y el pilar a través del macrodiseño del implante, torque, precarga, diseño y material del tornillo.

El sistema de conexión externa posee un dispositivo antirotacional, inicialmente la función de este dispositivo diseñado como hexagono era proporcionar un mecanismo de torque rotacional que asegure y facilite la transferencia del implante en su montura durante la coloca- ción quirúrgica en el hueso, la altura del hexágono era de $0,7 \mathrm{~mm}$ pero debido al aflojamiento o fractura del tornillo ante diversas fuerzas tensionales, algunos sistemas incrementaron la altura a 1,2 $\mathrm{mm}$ para obtener estabilidad y mantener un óptimo efecto antirotacional $(3,4)$. Por otra parte, la extensión hexagonal del implante, se utiliza como un dispositivo de orientación para la cofia de impresión (5). Algunos trabajos demostraron que una plataforma de implante con mayor diámetro resulta en una adecuada precarga a través de la interfase pilar-implante sin sobrecargar al tornillo protésico de fijación (6). 


\section{Revisión de literatura}

La unión de los componentes protésicos al implante se da por medio de tornillos de titanio u oro, es importante que los tornillos se ajusten de acuerdo a las especificaciones del fabricante. Los tornillos presentan diferentes propiedades mecánicas de acuerdo a su tamaño, diseño y composición metalúrgica, debiendo ajustarse de 50 a $75 \%$ de $\mathrm{su}$ resistencia para proporcionar una óptima fuerza de unión (7).

La falta de adaptación entre el pilar y la plataforma del implante origina ejes de fulcrum produciendo tensión a nivel de la interfase pilar e implante. En una adaptación óptima entre la plataforma del implante y el pilar, se crean puntos de fulcrum continuos alrededor de la circunferencia. Cuando hay estabilidad, las fuerzas oclusales verticales no ejercen tensión sobre el tornillo, ni causan su aflojamiento o fractura, esto no es válido cuando se atornillan pilares no maquinados sobre la plataforma del implante produciendo gaps verticales y horizontales, la carga vertical puede causar compresión de los pilares no maquinados y aflojamiento o pérdida del tornillo. En cualquier situación cuando la fuerza se aplica fuera del eje de fulcrum o de rotación al inicio de la carga, ésta es de magnitud suficiente como para superar la fuerza de sujeción, resultando en estiramiento, aflojamiento o fractura del tornillo. Esta fuerza desfavorable puede evitarse en su componente vertical y horizontal, para mantener el equilibrio el momento de resistencia del tornillo debe ser igual o mayor que la suma de los momentos creados por la fuerza que se aplica fuera del eje de rotación (7).
Segundo y col. (8) realizaron un estudio de elementos finitos para comprobar la distribución de las fuerzas en implantes de hexágono externo, pilares, tornillos (Gold Tite) y coronas en la región posterior. El análisis fue realizado con un modelo virtual 3D recreando una precarga de $35 \mathrm{~N} / \mathrm{cm} 2$ aplicada a la interfase pilar- implante (3i, $4,0 \times 11,5 \mathrm{~mm})$. La carga aplicada fue de $382 \mathrm{~N} / \mathrm{cm} 2$ a $6 \mathrm{~mm}$ del centro del implante con una angulación de $15^{\circ}$. Se encontró que la mayor carga se producía en la interfaz entre la plataforma del implante y el pilar, y en el punto medio del diámetro interno de la primera rosca del tornillo, sobre el lado de aplicación de carga. Se observó cómo estas fuerzas podrían llegar a deformar el hexágono y girar la corona.

Desde el punto de vista biomecánico, dos factores importantes podrían ser descritos como principales elementos de estabilidad de unión implante-pilar en el sistema de hexágono externo: precarga y dispositivo antirotacional (9). El torque es el movimiento producido por la aplicación de una fuerza tangencial al tornillo y es igual a la fuerza multiplicada por la distancia del punto de aplicación al centro de rotación del objeto. Se expresa en Newtons por centímetro cuadrado (N/cm2) (10). La magnitud del torque aplicado está limitada por la resistencia a la elasticidad del tornillo y la resistencia de la interfase hueso -implante es el límite biológico del torque (11). La precarga es definida como la fuerza de tensión que se acumula desde la cabeza hasta las roscas del tornillo como producto del ajuste del tornillo, además la precarga depende principalmente de la torsión aplicada y en segundo lugar del material, diseño de la cabeza, roscas del tornillo y de la rugosidad de la superficie (1). La precarga adecuada es obtenida por un torque óptimo y está limitada por la resistencia a la fricción de la rosca del tornillo en contacto y se opone a la superficie de contacto, esta fuerza de tensión une el pilar contra el implante o la cofia contra el pilar $(12,13)$. De acuerdo con esta teoría, la precarga previene la separación de los componentes del implante, protegiendo así al tornillo contra fracturas y distribuyendo la tensión. Si la precarga óptima protege al tornillo, la precarga inadecuada podría causar que los tornillos protésicos se volvieran más susceptibles a fracturarse en funcionamiento. Las cargas funcionales aplicadas a una prótesis sobre implante son compresivas, aunque el brazo de palanca sea muy corto para el implante, el momento de tensión está presente excepto cuando la carga está directamente centrada sobre y paralela al implante. La conexión de hexágono externo no está diseñada para soportar cargas de tensión si esto sucediera la tensión estaría soportada por el tornillo protésico de retención (12).

Motash informó que sólo el 10\% del torque aplicado al ajuste inicial de un sistema de rosca se mantiene para inducir la precarga mientras que el $90 \%$ sirve para superar la fricción entre los componentes de acoplamiento (14). Por otra parte encontró que las fuerzas cíclicas que se producen al momento de ajustar y aflojar el tornillo disminuyen la fricción entre las roscas durante la aplicación del torque, esto se debe al desgaste de la microrugosidades entre las superficies de contacto, en consecuencia aumenta la precarga axial. Cibirka y col. determinaron que la estabilidad de 
unión del tornillo involucra 3 factores: adecuada precarga, ajuste pasivo y la característica antirotacional de la interfase implante-pilar (15). Al ajustar el tornillo del pilar se genera una fuerza compresiva que mantiene unida la plataforma del implante y el pilar. Si el tornillo se desajusta y la precarga desciende bajo un nivel crítico, la estabilidad será comprometida y clínicamente potencia el fracaso. Se podría asumir que por el contrario, sin la precarga, el tornillo tendría que soportar toda la carga aplicada en la unión, lo cual acortaría la vida de fatiga del tornillo (16). Muchos estudios se han ocupado de los mecanismos de ensamblaje e informaron que una interfase incorrecta da lugar a excesivo estrés en la unión del tornillo del pilar, produciendo inestabilidad. Patterson y col. determinaron que los componentes prefabricados como cilindros de oro unidos al pilar, protegen al tornillo del pilar de los efectos de las cargas aplicadas (17). Andersen y cols. determinaron que un implante con una interfase de diámetro ancho experimenta menos aflojamiento de tornillo que un implante con una interfase de diámetro estándar o angosto cuando es sujeto a diversas fuerzas (6). Cuando una prótesis sobre implante de diámetro ancho es sujeta a fuerzas masticatorias, las fuerzas transmitidas a la interfase entre el pilar y el cuerpo del implante son distribuidas a lo largo de un área de superficie más grande (un implante de $6,0 \mathrm{~mm}$ de diámetro tiene $256 \%$ más área de superficie de plataforma en la interfase que un implante de $3,75 \mathrm{~mm}$ diámetro). Con menos deformación plástica en la interfase, hay menos deformación de tornillo y micromovimiento a ese nivel.
Factores que afectan la reducción de precarga sobre el tornillo del pilar

El problema de desprendimiento del tornillo fue atribuido generalmente a la complejidad de la condiciones de la carga masticatoria, ya que pueden inducir complejas tensiones en la restauración del implante (18). Los posibles factores causales que afectan la reducción de la precarga sobre el tornillo y por lo tanto la inestabilidad de la unión del tornillo son:

a. Sobrecarga de flexión. La flexión es una situación de carga crítica que puede producir la inestabilidad del tornillo. Una fuerza de flexión más larga que la resistencia a la flexión de los tornillos resulta en una deformación plástica que permite la perdida de la precarga. El punto de flexión de un tornillo de oro es $1370 \mathrm{~N}$, calculado de acuerdo a las dimensiones del tornillo y especificaciones del material (19).

b. Fatiga. Es la propagación de grietas progresivas que finalmente se traduce en una fractura bajo cargas repetidas por debajo del límite de elasticidad (20). La fatiga dinámica en los implantes dentales ocurre cuando la carga cíclica es aplicada al sistema en un nivel por debajo de la resistencia a la elasticidad del material del tornillo del pilar. Versuils y col. reportaron que el tornillo del pilar podría desajustarse o fracturarse cuando es fatigado o sobrecargado (21).

c. Relajación del asentamiento. Es el desgaste de las rugosidades microscópicas en las superficies de contacto, causado por el micromovimiento cuando la unión está sujeta a cargas y vibraciones externas. Este efecto está basado en que las superficies no son totalmente lisas y que las superficies maquinadas exhiben algún grado de microrugosidades. El desgaste en el área de contacto puede llevar a las 2 superficies a estar más cerca una de la otra, por lo tanto cuando el efecto total del asentamiento excede la elongación elástica del tornillo, el tornillo se afloja debido a la perdida de tensión en el cuello y la precarga cesa bajo el cuello y las roscas. Por esta razón se recomienda que el tornillo del pilar sea reajustado después de la inserción inicial y periódicamente cada vez que sea posible para verificar el ajuste apropiado $(21,22)$. Se estimó que el 2 al 10\% de la precarga inicial se pierde debido al efecto de asentamiento, por consiguiente se requiere un torque menor para el desajuste del tornillo (comparado con el torque inicial de ajuste) (23).

d. Micromovimiento. Trabajos de investigación reportaron que los micromovimientos causados por fuerzas de cizallamiento son responsables del aflojamiento del tornillo. El movimiento vibratorio flexiona el tornillo, el cual causa un desajuste o pérdida de contacto entre las roscas del tornillo y las roscas interna del implante, así como la superficie de la cabeza del tornillo y del cuerpo del pilar (24).

\section{Tornillos del pilar y protésicos}

Son dispositivos que afrontan las interfases del pilar a la plataforma del implante, de la cofia al pilar 
y entre ambos tornillos. El factor más importante que determina las características de los tornillos roscados son las propiedades del material y los fabricantes han hecho numerosos cambios en ese sentido. La resistencia a la fricción entre el titanio de las roscas del implante y la rosca del tornillo, resultan en una forma de desgaste adhesivo que se produce durante el íntimo contacto de deslizamiento de los 2 materiales, limitando la precarga de las características del tornillos de titanio. Por lo tanto la transición se ha hecho con el tornillo de aleación de oro (25). Bickford describió el proceso de desajuste del tornillo en 2 etapas: Etapa inicial, en la cual las fuerzas externas (masticación) aplicadas a los tornillos conectados causan un deslizamiento de las roscas contribuyendo a la liberación de la precarga de los tornillos que se relaciona con la aplicación de fuerzas transversales y axiales que son los suficientemente grandes para superar las fuerzas de fricción y compresión que mantienen las superficies de contacto en una relación fija. Además describió que los tornillos con una extensa elasticidad por la precarga son mantenidos por la fricción adecuada de las roscas (26). Las fuerzas externas pueden crear un movimiento vibratorio y causar el retiro de las roscas produciendo reducción en la efectividad de la precarga. Una pequeña escala de desajuste, como una desalineación del tornillo podría afectar la precarga. La segunda etapa compromete continuamente la reducción de la precarga bajo un nivel crítico a través de la fuerza externa y micro-movimiento, permitiendo que las roscas giren y pierda la función del tornillo de conexión. Binon y col. describieron que la relación entre el torque aplicado al tornillo y la precarga es afectada por muchas variables, incluyendo las propiedades del material, diámetro, geometría de la configuración y coeficiente de fricción entre las 2 superficies en contacto, rigidez de los hilos, acabados de superficie, propiedades y cantidad de lubricante y la velocidad de ajuste del tornillo (4). Clínicamente, muchas variables adicionales pueden afectar el procedimiento de ajuste del tornillo. Variaciones en la aplicación del torque, la técnica del operador, la presencia de fluidos orales, la velocidad de ajuste, y el uso de ajuste manual antes que el ajuste con torquímetro son posibles fuentes de variación en el alcance de óptima precarga.

Pauleto concluyó que el tornillo protésico de titanio demostró evidencias de desajuste en un $50 \%$ de las restauraciones durante el primer año en función, un fenómeno que también fue observado en $43 \%$ de implantes unitarios (27). Otros estudios clínicos reportaron que el aflojamiento y/o fractura del tornillo disminuyó significativamente cuando se utilizó un tornillo de aleación de oro en el pilar CeraOne Esto fue atribuido a la mayor cantidad de fuerza de fricción producidas entre el tornillo de aleación de oro y el componente del implante $(25,28)$. Zarb y Schmitt reportaron que los tornillos protésicos de oro poseen menor coeficiente de fricción, son ajustados efectivamente para obtener mayores precargas, no se adhieren al titanio, pueden lograr precargas de más de $890 \mathrm{~N}$ y su límite de elasticidad es de $75 \%$ obteniendo valores de 1270 a 1 $380 \mathrm{~N}$ siendo más del doble que los tornillos de titanio (29). El tornillo de titanio posee mayor módulo de elasticidad y mayor rigidez, concentra las fuerzas y no las transmite adecuadamente.

\section{Dispositivo antirotacional}

Es indispensable que se cuente con un mecanismo antirotacional que dará estabilidad y le dará la posibilidad de reposicionamiento (30). Cuando el implante es rehabilitado comenzará a recibir cargas, si las condiciones no son favorables un efecto secundario resulta en micromovimientos. El micromovimiento en el sistema lleva a rupturas en el material, en la superficie del implante y en las interfase de la unión del tornillo e implante, produciendo la pérdida de precarga, que resulta en reducidas fuerzas de contacto entre el pilar y la plataforma del implante, de este modo, se acelera el aflojamiento de la unión del tornillo (24).

En una evaluación de la precisión y la consistencia del maquinado, Binon reportó que el ajuste del hexágono implante-pilar es importante en restauraciones individuales "donde el asentamiento exacto es crítico para conseguir contactos interproximales reproducibles y características antirotacionales óptimas" (31). Se describió que la tolerancia del maquinado de la presente tecnología alcanzó 3 a 5 um con tornillos maquinados controlados numéricamente mediante computador.

\section{Recomendaciones para rehabili- tación implanto-soportada}

En la prótesis sobre implante, cuando se adopta un sistema hexagonal clásico, algunas reglas son fundamentales en la elaboración de coronas protésicas como ajuste pasivo, ferulización, polígono de estabilización, tabla oclusal reducida en sentido bucolingual y cúspides bajas (32). 


\section{a. Ajuste pasivo.}

Es el contacto máximo entre la base de la infraestructura metálica y los pilares intermediarios, sin generar tensión entre estos pilares (33). Las posibles complicaciones del ajuste no pasivo de las estructuras pueden ser categorizadas en 2 grupos: 1) complicaciones biológicas que incrementan la transferencia de carga al hueso, pérdida del hueso y desarrollo de la microflora en el gap entre el implante y el pilar y, 2) complicaciones protésicas: aflojamiento o fractura del tornillo y fractura del implante (34).

La fuerza de momento suele actuar en la interfase implante-pilar y se puede traducir en fractura y desprendimiento del tornillo, esto es más común en implantes con conexión externa (35). Por eso la necesidad de la elaboración de superestructuras con ajuste pasivo y función antirotaciónal al mismo tiempo (32). La fabricación de la prótesis sobre implante requiere de muchos procedimientos clínicos y de laboratorio que deben ser muy precisos. La posible distorsión de la prótesis puede ocurrir durante la toma de impresión, durante la fabricación del modelo maestro, durante la elaboración del patrón de cera, durante el revestimiento y procedimientos de colado y durante la cocción de la porcelana (34).

\section{a.1. Durante el procedimiento de} impresión: La transferencia en prótesis sobre implante es la más precisa que existe, dado que los elementos disponibles para este procedimiento y el implante permanecen clínicamente inmóviles. La característica de pasividad de las estructuras protésicas es influenciada por detalles de la transferencia y de los elementos relacionados (32). Cualquier inexactitud dimensional en este proceso conducirá a un resultado comprometido $\mathrm{y}$, posiblemente, el fracaso del tratamiento, por lo tanto, la técnica de transferencia es una etapa crítica en este proceso (36). Las copas de transferencia ferulizadas o no ferulizadas con resina acrílica presentan controversia. Assif y cols. encontraron que el método con copas de transferencia ferulizadas producen el menor error, el cual tiene diferencia estadísticamente significativa con respecto al método sin ferulización (37). Por el contrario, Phillips y cols. no encontraron diferencia significativa entre los 2 métodos de impresión (38). En cuanto a la estabilidad dimensional del material de impresión, los materiales de impresión como polivinilsiloxano y polieter son apropiados para la toma de impresión de implantes (38).

a.2. Durante la fabricación del modelo maestro: $\mathrm{La}$ exactitud de un modelo maestro para tratamiento de implantes depende del tipo de material de impresión, la técnica de impresión de los implantes, precisión del material modelo y técnica de modelo maestro (39). La expansión de fraguado del yeso tipo IV usado para la fabricación del modelo maestro es de $0,1 \%$ mientras que el yeso tipo $\mathrm{V}$ tiene una expansión de fraguado de $0,3 \%$ que compensa la contracción de la solidifación de la aleación metálica (40).

a.3. Durante la elaboración del patrón de cera (distorsión):
La cera tiene el mayor coeficiente de expansión térmica entre los materiales dentales, y su estabilidad dimensional depende de los cambios de temperatura del aire. Los cambios dimensionales pueden producir una pobre adaptación en el modelo si no es balanceado mediante factores de compensación de la expansión del modelo. La contracción de la cera en el paso de estado líquido a sólido puede ser de $0,4 \%$. Adicionalmente el patrón tiende a liberar tensiones que fueron incorporadas durante el manejo de la cera debido a un calentamiento no uniforme (41).

a.4. Durante el revestimiento y procedimientos de colado: Expansión del revestimiento: los revestimientos son de alta combustión y unión al fosfato, presentan una expansión de fraguado que va entre $0,23 \mathrm{y}$ $0,50 \%$. Su expansión hidroscópica va de $0,35 \%$ a $1,20 \%$ y la expansión térmica de 1,33 a $1,58 \%\left(700^{\circ} \mathrm{C}\right)(41)$.

Contracción del metal: se ha demostrado que la contracción del metal ocurre en 3 etapas: 1) contracción térmica del metal en estado líquido entre la temperatura a la cual es calentado y la temperatura líquida, 2) contracción del metal inherente a su cambio del estado líquido al sólido y 3) contracción térmica del metal sólido que ocurre hasta alcanzar la temperatura ambiente. La contracción térmica de las aleaciones dentales pueden ir de $1,42 \%$ en un tipo III a $1,56 \%$ en un tipo I (42).

\section{a.5. Durante la cocción de la por-} celana: La distorsión ocurre en el cuerpo de la estructura metálica curva y extensa de la 
prótesis parcial fija. Se ha demostrado que esta distorsión es el resultado de cambios en el metal así como la contracción de la porcelana, y esto ocurre principalmente durante la etapa de desgasificación y del glaseado final del ciclo de cocción de la porcelana (43).

\section{b. Oclusión}

La oclusión es otro factor que interviene en la selección del tipo de restauración atornillada o cementada. Idealmente, en el caso de dientes posteriores, el implante debería ser colocado en la fosa central para generar una carga axial. La dimensión bucolingual de premolares superiores es de $9 \mathrm{~mm}$ aproximadamente, mientras que los primeros $\mathrm{y}$ segundos molares superiores es de $11 \mathrm{~mm}$ (34). La tabla oclusal de los dientes mencionados es de 4,5 en premolares y 5 - $6 \mathrm{~mm}$ en molares. La cabeza de los tornillos de fijación tiene un diámetro de $3 \mathrm{~mm}$, por lo tanto requiere como mínimo un orificio de acceso de $3 \mathrm{~mm}$ de diámetro, estos $3 \mathrm{~mm}$ representan el $50 \%$ de la tabla oclusal de las molares y más del 50\% de la tabla oclusal de las premolares (7). Para establecer contactos oclusales adecuados, estos deben ser realizados sobre resina, la cual es usada generalmente para cubrir los orificios de los tornillos (34). Este orificio se encuentra en una zona de riesgo de fractura de la porcelana, puesto que no hay soporte en los bordes del material de la infraestructura. Por otra parte Arita concluyó que el área del orificio oclusal ocupa alrededor del 33\% de la superficie funcional de un diente posterior (32). Por el contrario con prótesis cementada los contactos oclusales ideales pueden ser establecidos y permanecer estables por un largo periodo (34).

En una corona sobre implante, en la región de la primer molar, el área de contacto oclusal en fosa seria descartada en el caso de una prótesis atornillada. Por otra parte, la compensación por el desplazamiento del contacto oclusal de la cúspide vestibular debe ser evitada, puede generar una carga fuera del centro del implante, lo que permite la formación de una fuerza de momento (32).

La sobrecarga oclusal, posiblemente se da como resultado de un cantiliver amplio $(>15 \mathrm{~mm}$ en la mandíbula y $>10-12 \mathrm{~mm}$ en el maxilar), exceso de contactos prematuros ( $>100 \mu \mathrm{m})$, actividades parafuncionales, inadecuado diseño oclusal, inadecuado número de implantes, tabla oclusal larga y pobre densidad ósea. Estos factores pueden ser limitantes para la longevidad del implante. Por lo tanto, es importante controlar la oclusión del implante dentro de los límites fisiológicos y por ello suministrar una carga óptima para asegurar un éxito del implante a largo plazo (44).

Para obtener éxito del implante a largo plazo se debe tonar en consideración la oclusión dependiendo el caso:

- Consideraciones oclusales para arcada completa de prótesis fija: Oclusión bilateral balanceada con la oposición de una prótesis completa, oclusión en función de grupo u oclusión mutuamente protegida con deslizamiento de la guía anterior al oponerse a la dentición natural, infraoclusión en el segmento del cantiliver $(100 \mu)$ y Deslizamiento en céntrica $(1-1,5 \mathrm{~mm})$
- Consideraciones oclusales en sobredentadura: Oclusión balanceada bilateral empleando oclusión lingualizada y oclusión con dientes monoplano sobre reborde severamente reabsorbido

- Consideraciones oclusales en prótesis fija sector posterior: Guía anterior con dentición natural, oclusión en función en grupo comprometiendo los caninos, contactos céntricos, tabla oclusal reducida, cúspides planas, minino cantilever y oclusión posterior mordida cruzada cuando sea necesaria

- Consideraciones oclusales en prótesis fija única: Guía anterior o lateral con la dentición natural, contacto ligero ante una mordida intensa y sin contacto ante una mordida suave, deslizamiento en céntrica (1-1,5mm área plana) y aumento de los contactos proximales (44).

\section{c. Polígono de estabilización}

La disposición no lineal de los implantes en la colocación quirúrgica es beneficiosa, ya que se puede generar un polígono para otorgar mayor estabilidad (32).

Huang y cols. evaluaron los efectos biomecánicos al colocar implantes alineados o escalonados en una prótesis fija, determinaron que la configuración escalonada disminuye el estrés en los implantes y que la distribución del estrés a nivel óseo no fue significativamente diferente tanto en la configuración escalonada como en la alineada (45). Por el contrario, Sato y cols. determinaron que la disposición escalonada no compensa el estrés producido por cargas laterales, sin embargo la utilización de un implante ancho y la disminución de la inclinación cuspídea sí disminuye 
el estrés y por lo tanto estos deben ser considerados antes que la configuración escalonada (46). Akça y cols. determinaron que un sistema escalonado requiere un ancho buco lingual adecuado (47), por lo que el uso de implantes anchos es más viable, fácil y mejora la disipación del estrés.

\section{Conclusiones}

1. La estabilidad de unión de la plataforma del implante y el pilar en el sistema de conexión externa desde el punto de vista mecánico se da por elementos antirotacionales y una adecuada precarga.

2. La falta de precisión y/o estabilidad entre el implante/pilar pueden producir diversas complicaciones: aflojamiento o fractura del tornillo del implante, fatiga o fractura del implante, pérdida ósea marginal y fractura ósea.

3. En prótesis sobre implantes la ausencia de ajuste pasivo en la estructura protésica, la carga excesiva de la prótesis en relación con la longitud y el número de implantes, los hábitos parafuncionales y las discrepancias oclusales traen diversas complicaciones como aflojamiento o fractura del tornillo del pilar.

\section{Referencias Bibliograficas}

1. Branemark PI, Hansson BO, Adell R, Breine U, Lindstrom $\mathrm{J}$, Hallén O. Osseointegrated implants in the treatment of the edentulous jaw: Experience from a 10 -year period. Scand J Plast Reconst Surg Suppl. 1977;16:1-132.

2. Sones AD. Complications with osseointegrated implants. J Prosthet Dent. 1989;62(5):5815.

3. English CE. Externally hexed implants, abutments, and transfer devices: a comprehensive overview. Implant Dent. 1992;1(4): 273-83.

4. Binon PP. Implants and components: entering the new millennium. Int J Oral Maxillofac Implants. 2000;15(1):76-94.

5. Fenton AH, Zarb GA. Research status of prosthodontics procedures. Int J Prosthodont. 1993; 6(2):137-44.

6. Andersen E, Saxegaard E, Knutsen BM, Haanaes HR. A prospective clinical study evaluating the safety and effectiveness of narrow-diameter threaded implants in the anterior region of the maxilla. Int J Oral Maxillofac Implants. 2001;16(2): 217 24.

7. Hebel KS, Gajjar RC. Cementretained versus screw-retained implant restorations: achieving optimal occlusion and esthetics in implant dentistry. J Prosthet Dent. 1997;77(1): 28-35.

8. Segundo RM, Oshima H, Silva I, Junior L, Mota E, Coelho L. Stress distribution on external hexagon implant sistem using 3D element analysis. Acta Odontolog Latinoamericana. 2007; 20(2): 79-81.

9. Khraisat A, Abu-Hammad O, AlKayed AM, Dar-Odeh N. Stability of the implant/abutment joint in a single-tooth externalhexagon implant system: clinical and mechanical review. Clin Implant Dent Relat Res. 2004; 6(4): 222-29.

10.Misch CE. Contemporary implant dentistry. 3rd ed. St Louis: Mosby; 2007.

11.Tjellstrom A, Jacobson M, Albrektsson T. Removal torque of osseointegrated craniofacial implants: a clinical study. Int J Oral Maxillofac Implants. 1988; 3(4): 287-9.
12.Jaarda MJ, Razzoog ME, Gratton DG. Geometric comparison of five interchangeable implant prosthetic retaining screws. J Prosthet Dent. 1995;74(4): 373-9.

13. Cantwell A, Hobkirk JA. Preload loss in gold prosthesis-retaining screws as a function of time. Int J Oral Maxillofac Implants. 2004;19(1):124-32.

14. Motash N. Development of design charts for bolt pre-loaded up to the plastic range. J Eng Ind. 1976; 98: 849-851.

15.Cibirka RM, Nelson SK, Lang BR, Rueggeberg FA. Examination of the implant-abutment interface after fatigue testing. J Prosthet Dent. 2001; 85(3): 26875.

16.Binon P, Sutter F, Beaty K, et al. The role of screws in implant systems. Int J Oral Maxillofac Implants. 1994; 9: 48-63.

17.Patterson EA, Johns RB. Theoretical analysis of the fatigue life of fixture screws in osseointegrated dental implants. Int J Oral Maxillofac Implants. 1992; 7(1): 26-33.

18.Timmer J, Lauk M, Pfleger W, Deuschl G. Gross spectral analysis of physiological tremor and muscle activity. II. Application to synchronized electromyogram. Biol Cybern. 1998;78(5):359-68.

19.Jornéus L, Jemt T, Carlsson L. Loads and designs of screw joints for single crowns supported by osseointegrated implants. Int J Oral Maxillofac Implants. 1992;7(3): 353-59.

20.Craig RG, Powers JM. Restorative dental materials. 11th ed. St. Louis: Mosby; 2002.

21.Versulis A, Korioth TW, Cardoso AC. Numerical analysis of a dental implant system 
preloaded with a washer. Int J Oral Maxillofac Implants. 1999;14(3):337-41.

22. Sakaguchi RL, Borgersen SE. Nonlinear contact analysis of preload in dental implant screws. Int J Oral Maxillofac Implants. 1995;10(3): 295-302.

23. Shigley JE, Mischke CR. Standard handbook of machine design. 1st ed. New York: McGraw-Hill; 1986. p. 23-26.

24. AbuyoussefH, Weiner S, Ehrenberg D. Effect of an antirotation resistance form on screw loosening for single implant-supported crowns. J Prosthet Dent. 2000; 83(4):450-55.

25.Andersson B, Odman P, Lindvall AM, Branemark PI. Cemented single crowns on osseointegrated implants after 5 years: results from a prospective study on CeraOne. Int J Prosthodont. 1998;11(3):212-8.

26.Bickford JH. An introduction to the design and behavior of bolted joints. New York: Marcel Dekker; 1995.p.515-564.

27.Pauletto N, Lahiffe BJ, Walton JN. Complications associated with excess cement around crowns on osseointegrated implants. Int J Oral Maxillofac Implants.1999;14(6): 865-8.

28.Scheller H, Urgell JP, Kultje $\mathrm{C}$, et al. A 5-year multicenter study on implant-supported single crown restorations. Int J Oral Maxillofac Implants. 1998;13(2): 212-8.

29.Zarb GA, Schmitt A. The longitudinal clinical effectiveness of osseointegrated dental implants: the toronto study. Part III: problems and complications encountered. J Prosthet Dent.1990; 64(23): 185-94.

30. Wiskott HW, Jaquet R, Screrrer SS, Belser UC. Resistance of in- ternal-connection implant connectors under rotational fatigue loading. Int J Oral Maxillofac Implants. 2007; 22(2): 249-57.

31.Binon PP. Evaluation of machining accuracy and consistency of selected implants, standard abutments and laboratory analogs. Int J Prosthodont. 1995; 8(2):162-78.

32.Arita C. Prótese sobreimplantes no segmento posterior. Rev Implant News. 2006; 3(4):336-43.

33. Dinato JC. Implantes oseointegrados cirugia y protesis. 1era ed. Sao Paulo: Artes Medica; 2003.

34. Michalakis KX, Hirayama $\mathrm{H}$, Garefis PD. Cemented-retained versus screw-reteined implant restorations: a critical review. Int J Oral Maxillofac Implants. 2003;18(5):719-28.

35.Celletti R, Pameijer $\mathrm{CH}$, Bracchetti G, Donath K, Persichetti G, Visani I. Histologic evaluation of osseointegrated implants restored in nonaxial functional occlusion with preangled abutments. Int $\mathrm{J}$ Periodontics Restorative Dent. 1995;15(6):562-73.

36.Phillips KM, Nicholls JI, Ma $\mathrm{T}$, Rubenstein J. The accuracy of three implant impression techniques: a three-dimensional analysis. Int J Oral Maxillofac Implants. 1994; 9: 533-540.

37. Assif D, Marshak B, Schmidt A. Accuracy of implant impression techniques. Int J Oral Maxillofac Implants. 1996;11(2):21622.

38.Daoudi MF, Setchell DJ, Searson LJ. A laboratory investigation of the accuracy of two impression techniques for single-tooth implants. Int $\mathrm{J}$ Prosthodont. 2001;14(2):152-8. 39.Del'Acqua M, Arioli-Filho JN,
Compagnoni MA, Mollo Fde A Jr. Accuracy of impression and pouring techniques for an implant-supported prosthesis. Int J Oral Maxillofac Implants. 2008;23(2):226-36.

40.Phillips RW. Skinner's science of dental materials. Philadelphia: Saunders; 1991.

41.O'Brien WJ. Dental materials and their selection. Chicago: Quintessence; 1997. p.147-150.

42.Craig RG. Restorative dental materials. 10 edition. St Louis: Mosby; 1997.

43.Bridger DV, Nicholls JI. Distortion of ceramometal fixed partial dentures during the firing cycle. J Prosthet Dent. 1981;45(5): 507-14.

44.Kim Y, Oh TJ, Misch CE, Wang HL. Occlusal considerations in implant therapy: clinical guidelines with biomechanical rationale. Clin Oral Implants Res. 2005;16(1): 26-35.

45.Huang $\mathrm{Hl}$, Lin $\mathrm{Cl}$, Ko CC, Chang CH, Hsu JT, Huang JS. Stress analysis of implant-supported partial prostheses in anisotropic mandibular bone: inline versus offset placements of implants. J Oral Rehabil. 2006; 33(7): 501-8.

46.Sato Y, Shindoi R, Hosokawa R, Tsuga, Akagawa Y. A biomechanical effect of wide implant placement and offset placement of three implants in the posterior partially edentulous region. J Oral Rehabil. 2000; 27(1):1521.

47.Akça K, Iplikçioğlu H. Finite element stress analysis of the influence of staggered versus straight placement of dental implants. Int J Oral Maxillofac Implants. 2001;16(5):722-30. 